\title{
Cutaneous leucocytoclastic vasculitis associated with acute sarcoidosis
}

\author{
C. JOHNSTON \\ M.A., M.R.C.P. \\ C. KENNEDY* \\ M.A., M.R.C.P. \\ Department of Communicable Diseases, St George's Hospital, Blackshaw Road, London, SW17 0QT
}

\begin{abstract}
Summary
A case of acute sarcoidosis associated with the simultaneous appearance of cutaneous leucocytoclastic vasculitis and erythema nodosum is reported. Circulating immune complexes were demonstrated and may have been important in the pathogenesis of both types of skin lesion.
\end{abstract}

KEY WORDS: erythema nodosum, immune complexes.

\section{Introduction}

Cutaneous involvement in sarcoidosis is said to occur in about 15\% of cases (Scadding, 1967). With the exception of erythema nodosum, all the clinical patterns histologically show non-caseating granulomata. The case we report demonstrates the simultaneous occurrence of leucocytoclastic vasculitis and erythema nodosum. The presence of circulating immune complexes was demonstrated and may have been important in the pathogenesis of both types of skin lesion.

\section{Case report}

A 41-year-old male presented with a 3-week history of cough, pyrexia, arthralgia of the knees, arthritis of both ankles and tender erythematous nodules over the left tibia. Abnormal investigations at this stage were an ESR of $41 \mathrm{~mm}$ in $1 \mathrm{hr}$ and bilateral broncho-pulmonary lymphadenopathy on chest X-ray. Mantoux testing at 1:100 was negative.

For the next 2 weeks he remained systematically unwell with increasing arthritis. In addition to bilateral erythema nodosum he developed discrete, non-tender, slightly raised, purplish plaques situated over the dorsal aspects of both forearms and on the right side of his neck. There was bilateral conjunctivitis but no uveitis.

Investigations now showed an ESR of $80 \mathrm{~mm}$ in 1 hr; bilirubin was 14 iu/l, alkaline phosphatase 128

*Present address: Southmead Hospital, Westbury-on-Trym, Bristol, BS10 5NB. $\mathrm{iu} / 1$ (normal 20-100), alanine transaminase $38 \mathrm{iu} / 1$ (normal 10-55), albumin $28 \mathrm{~g} / 1$ (normal 35-45). $\alpha 1$ and $\alpha 2$ globulins were elevated but total immunoglobulins were normal. Lung function tests showed a slight restrictive defect only; elecrocardiogram, renal function, serum and urinary calcium were normal. Complement levels were within the normal range at $\mathrm{CH}_{50} 110 \%$ and $\mathrm{C}_{3} 115 \%$ of normal. Circulating immune complexes were demonstrated by a Clq precipitation technique. The Kveim test was positive.

Skin biopsy from a purple plaque on the forearm showed a superficial peri-vascular mixed inflammatory cell infiltrate composed of lymphocytes with an admixture of neutrophils and nuclear dust (Fig. 1). Some vessels showed fibrinoid necrosis. There were no non-caseating granulomata.

The erythema nodosum and cutaneous vasculitis spontaneously resolved over the next 4 weeks, but 1 month later he had a recurrence of the cough, pyrexia and a fresh outbreak of cutaneous vasculitis on the upper limbs and spreading on to the trunk. He was treated with prednisolone ( $20 \mathrm{mg}$ daily) and the rash resolved within $48 \mathrm{hr}$. The prednisolone was discontinued over the next 4 weeks and he has subsequently remained asymptomatic.

\section{Discussion}

Vasculitis, in association with the non-caseating granulomata of sarcoidosis has been a recognized histological feature in the skin (Kennedy, 1979). Histological evidence of vasculitis has also been reported with an ulcerative form of erythroderma (Simpson, 1963) superficial to foci of non-caseating granulomata which the authors believed to be due to sarcoidosis (Chouvet et al., 1980) and in association with erythema annulare centrifugum-like lesions (Branford, Farr and Porter, 1982). In this report, leucocytoclastic vasculitis, i.e. evidence of vascular damage with an associated infiltrate of neutrophil polymorphs some of which are in a state of disintegration, was the main histological feature of the 


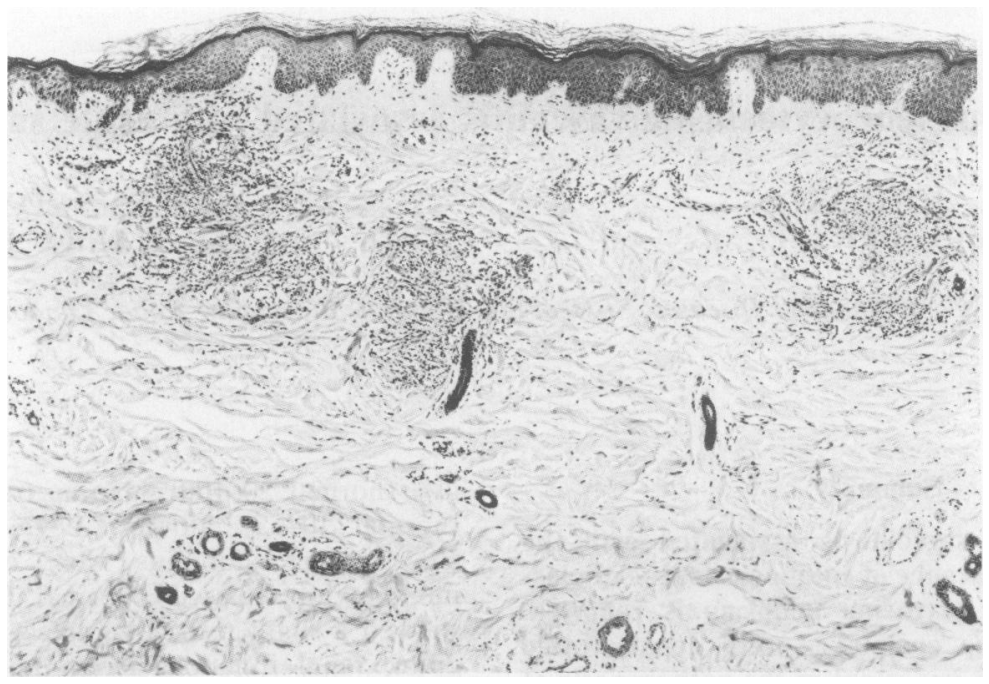

FIG. 1. Skin biopsy (H \& E $\times 54)$. Leucocytoclastic vasculitis in the superficial dermis.

cutaneous eruption associated with sarcoidosis. Noncaseating granulomata were not present.

The extra-pulmonary features of acute sarcoidosis (pyrexia, arthralgia, iritis and erythema nodosum) are believed to be mediated by circulating immune complexes. Such complexes can be found in the sera of most patients, provided a variety of techniques are used. The presence of granular deposits of immunoglobulin and complement within the lesions of erythema nodosum in association with demonstrable circulating immune complexes, suggests a causal relationship. Although we were unable to obtain a suitable specimen to stain for complement or immunoglobulins, the histology and presence of circulating immune complexes as demonstrated by $\mathrm{Clq}$ binding and the dramatic response to steroid therapy, suggests that like the erythema nodosum, these vasculitic skin lesions were also mediated by immune complexes.

\section{Acknowledgments}

The authors are grateful to Dr M. Wansborough-Jones fo: allowing us to study the patient and for his helpful comments.

\section{References}

BRANFORD, W., FARr, P. \& PORTER, D. (1982) Annular vasculition the head and neck in a patient with sarcoidosis. British Journatof Dermatology, 106, 713.

Chouvet, B., Del Grande, P., Enay, G., Perrot, H. \& THIVOLET, J. (1980) Vascularite leucocytoclastique et sarcoidose Apropos d'un cas. Annals of Dermatology and Venereology (Paris) $\mathbb{D}$ $107,279$.

KENNEDY, C. (1979) Sarcoidosis with cutaneous vasculitis. British $\overrightarrow{\overrightarrow{0}}$ Journal of Dermatology, 101, 47.

SCADDING, J.G. (1967) Sarcoidosis. p. 174. Eyre and Spottiswood, London.

SIMPSON, J.R. (1963) Sarcoidosis with erythroderma and ulceration: British Journal of Dermatology, 75, 193.

(Accepted 5 July 1983) 